\title{
ON THE SOBOLEV CLASS OF A COMPOSITE FUNCTION
}

\author{
DENNIS D. COX AND FINBARR O'SULLIVAN
}

(Communicated by Palle E. T. Jorgensen)

\begin{abstract}
It is shown that the Sobolev class of a function of the form $H(x, \theta(x))$ is the same as the Sobolev class of $\theta$, for sufficiently smooth $H$. This result has applications in a perturbation analysis of a nonlinear system of differential equations considered elsewhere.
\end{abstract}

\section{INTRODUCTION}

Here we give a result concerning the Sobolev class of a composite function. Specifically, we consider a function $f(x)=H(x, \theta(x))$ where $\theta$ is in a certain Sobolev space and $H$ is a smooth function in all of its arguments. We show that $f$ is in the same Sobolev space and derive a bound on its Sobolev norm. The need for such a result arose in the study of a discrete approximation to a nonlinear differential equation that arose in Cox and O'Sullivan [2]. See the discussion of the examples near the end of $\S 2.1$ of that paper. We would expect that such a result could have other applications in the study of nonlinear differential equations.

We now give some definitions and notation. For a normed linear space $V$, let $\|v \mid V\|$ denote the norm on $V$, and let $S(r, V)=\{v \in V:\|v \mid V\| \leq r\}$ denote the closed ball of radius $r$ centered at the origin. The Sobolev space of real-valued functions on a domain $\mathscr{X} \subset \mathbb{R}^{d}$ is denoted $W_{p}^{s}(\mathscr{X})$ as defined in Triebel [3]. Any of the various equivalent norms for $W_{p}^{s}(\mathscr{Z})$ may be used. A Sobolev space of $q$-dimensional vector-valued functions on $\mathscr{X}$ will be denoted $W_{p}^{s}\left(\mathscr{X} ; \mathbb{R}^{q}\right)$. We will also make use of $C^{m}\left(\mathscr{X} ; \mathbb{R}^{q}\right)$ where $m$ is an integer, which is the space of $m$ times continuously differentiable functions equipped with an appropriate supremum norm on derivatives.

Theorem 1. Let $\mathscr{X} \subset \mathbb{R}^{d}$ be a bounded, open, simply connected, nonempty set with $C^{\infty}$ boundary (see [3, 3.2.1.2]). Let $d / 2<s<(d+1) / 2$, and let $H: \mathscr{Z} \times \mathbb{R}^{q} \rightarrow \mathbb{R}$ be such that for all $R>0, H \in C^{k+1}\left(\mathscr{X} \times S\left(R, \mathbb{R}^{q}\right)\right)$, where $k$ is the largest integer less than $s$. If $\theta \in W_{2}^{s}\left(\mathscr{X} ; \mathbb{R}^{q}\right)$ and $\sup _{x \in \mathscr{L}}|\theta(x)| \leq R_{0}$,

Received by the editors February 8, 1993.

1991 Mathematics Subject Classification. Primary 46E35; Secondary 46N30.

Key words and phrases. Sobolev norm, composite function.

The first author's research was supported by the National Science Foundation under Grant No. DMS-9207730. The second author's research was supported by the National Institutes of Health under Grants No. CA-42593 and No. CA-42045. 
then $H(\cdot, \theta(\cdot)) \in W_{2}^{s}(\mathscr{Z})$ and $\left\|H(\cdot, \theta(\cdot)) \mid W_{2}^{s}(\mathscr{Z})\right\| \leq M\left[1+\left\|\theta \mid W_{2}^{s}\right\|\right]$ where $M$ is a constant depending on $\mathscr{Z}, H, s$, and $R_{0}$.

Remark. Note that $\theta \in W_{2}^{s}$ implies by Sobolev's embedding theorem [1, Theorem 5.4] (noting that our assumption of $C^{\infty}$ boundary for $\mathscr{Z}$ implies any of the cone conditions) that $\sup _{\mathscr{L}}|\theta|<\infty$.

Proof. Note that $s$ is not an integer. We will assume for now that $d>1$. The proof when $d=1$ is easier and will be indicated at the end. In this proof, we will write $M(a, b, \ldots)$ for a generic constant $M$ depending only on the quantities $a, b, \ldots$. Note that dependence on $\mathscr{Z}$ subsumes dependence on $d$ and dependence on $s$ subsumes dependence on $k$.

Using equation (1) of Theorem 4.4.2.1 of [3], a norm for the Besov space $B_{p q}^{s}(\mathscr{Z})(1<p, q<\infty, 0<r<\infty, r$ not an integer $)$ is

$$
\begin{aligned}
\left\|f\left|B_{p q}^{r}(\mathscr{X})\|=\| f\right| L_{p}(\mathscr{X})\right\|+\sum_{j=1}^{d} & \left\{\int_{|h| \leq \delta}|h|^{-q(r-k)-d}\right. \\
\times & {\left.\left[\int_{\mathscr{L}(h, \varepsilon, \tau)}\left|\Delta_{h} \frac{\partial^{k} f}{\partial x_{j}^{k}}(x)\right|^{p} d x\right]^{q / p} d h\right\}^{1 / q} }
\end{aligned}
$$

for any $\delta, \varepsilon, \tau$ sufficiently small, and $k$ the largest integer less than $r$. Here, $\Delta_{h} f(x)=f(x+h)-f(x)[3,2.5 .1$, p. 189] and

$$
\begin{aligned}
\mathscr{X}(h, \varepsilon, \tau)=\left(\mathscr{X} \backslash \mathscr{X}^{\tau}\right) & \cup\left\{z+(0, \tau] \nu_{z}: z \in \partial \mathscr{X} \text { and } 0 \leq \angle\left(h, \nu_{z}\right)<\varepsilon\right\}, \\
\mathscr{X}^{\tau} & =\left\{x \in \mathscr{X}: \inf _{z \in \mathscr{X}}|x-z|<\tau\right\}, \\
\nu_{z} & =\text { inner normal to } \partial \mathscr{X} \text { at } z, \\
\angle(h, \nu)= & \text { angle between } h, \nu \text { (always positive). }
\end{aligned}
$$

Since

$$
B_{p p}^{s} \cong W_{p}^{s},
$$

this gives an equivalent norm for the Sobolev spaces as well [3, Definition 4.2.1.1]. Here, $\cong$ denotes equality of two normed linear spaces up to equivalence of norms.

In order to denote partial derivatives of $H$, we will write the independent variables as $H(x, t), x \in \mathscr{Z}$ and $t \in \mathbb{R}^{q}$. Let $k$ be as in the statement of the theorem. For fixed $\theta \in W_{2}^{s}(\mathscr{X})$ let $f(x)=H(x, \theta(x))$. Using the chain rule and product rule we have

$$
\frac{\partial^{k} f}{\partial x_{j}^{k}}(x)=\frac{\partial^{k} H}{\partial x_{j}^{k}}(x, \theta(x))+\sum_{l} c_{l} \frac{\partial^{k_{0 l} H}}{\partial t_{l}^{k_{0 l}}}(x, \theta(x)) \prod_{i} \frac{\partial^{k_{i l}} \theta_{l}}{\partial x_{j}^{k_{i l}}}(x)
$$

where the $c_{l}$ 's are constants depending on $k, k_{0 l} \leq k$, all $k_{i l} \geq 1$, and

$$
\sum_{i \geq 1} k_{i l}=k \text {. }
$$

We do not indicate the upper limits on the indices $l$ and $i$ in (3) (the upper limit on $i$ depends on $l$ ). Suffice it to note that they are finite and depend only 
on $k$ and $q$. We have indicated through the subscripts that the $k_{i l}$ depend on $l$, but we will drop that dependence for convenience henceforth. We will assume for now that there is more than one summand in (4). The case of one summand will be easy to treat.

Using the hypothesis $H \in C^{k+1}\left(\mathscr{Z} \times S\left(R_{0}, \mathbb{R}^{q}\right)\right)$, it is easy to check that (5)

$$
\begin{aligned}
& \int_{|h| \leq \delta}|h|^{-2(s-k)-d} \int_{\mathscr{Q}(h, \varepsilon, \tau)} {\left[\frac{\partial H}{\partial x_{j}}(x+h, \theta(x+h))-\frac{\partial H}{\partial x_{j}}(x, \theta(x))\right]^{2} d x d h } \\
& \leq M\left(H, R_{0}\right)<\infty .
\end{aligned}
$$

Using boundedness of $\partial^{k_{0}} H / \partial t_{l}^{k_{0}}$ and Hölder's inequality, if $m$ is a fixed index, with the same range as $i$, then

$$
\begin{aligned}
& \int_{|h| \leq \delta}|h|^{-2(s-k)-d} \int_{\mathscr{X}(h, \varepsilon, \tau)}\left\{\frac{\partial^{k_{0}} H}{\partial t_{l}^{k_{0}}}(x+h, \theta(x+h))\left[\prod_{i<m} \frac{\partial^{k_{i}} \theta_{l}}{\partial x_{j}^{k_{i}}}(x+h)\right]\right. \\
& \times {\left.\left[\frac{\partial^{k_{m}} \theta_{l}}{\partial x_{j}^{k_{m}}}(x+h)-\frac{\partial^{k_{m}} \theta_{l}}{\partial x_{j}^{k_{m}}}(x)\right]\left[\prod_{i>m} \frac{\partial^{k_{i}} \theta_{l}}{\partial x_{j}^{k_{i}}}(x)\right]\right\}^{2} d x d h } \\
& \leq M\left(\mathscr{X}, H, R_{0}\right)\left\{\prod_{i \neq m}\left[\int_{\mathscr{Z}}\left|\frac{\partial^{k_{i}} \theta_{l}}{\partial x_{j}^{k_{i}}}(x)\right|^{2 q_{i}} d x\right]^{1 / q_{i}}\right\} \\
& \times \int_{|h| \leq \delta}|h|^{-2(s-k)-d}\left\{\int_{\mathscr{Z}(h, \varepsilon, \tau)}\left|\frac{\partial^{k_{m}} \theta_{l}}{\partial x_{j}^{k_{m}}}(x+h)-\frac{\partial^{k_{m}} \theta_{l}}{\partial x_{j}^{k_{m}}}(x)\right|^{2 q_{m}} d x\right\}^{1 / q_{m}} d h
\end{aligned}
$$

provided

$$
\sum_{i \geq 1} 1 / q_{i} \leq 1
$$

(Here, the $q_{i}$ depend on $l$ and $m$, but this will not be important, so we do not indicate this dependence.) It is necessary to check that each of the integrals on the r.h.s. of (6) is finite. By the embedding Theorem 4.6.1(c) [3, pp. 327-328]

$$
B_{p_{1}, q}^{r_{1}} \subset B_{p_{2}, q}^{r_{2}}
$$

provided

$$
1<p_{1} \leq p_{2}<\infty
$$

and

$$
r_{1}-d / p_{1} \geq r_{2}-d / p_{2}
$$

In (8), the embedding operator (inclusion map) is continuous, which means the space on the 1.h.s. has a stronger norm than the space on the r.h.s. Furthermore, by part (a) of the aforementioned theorem

$$
B_{q, p_{1}}^{r} \subset B_{q, p_{2}}^{r}
$$

provided

$$
p_{1} \leq p_{2},
$$


again with continuous embedding. We will use these results to show that the $q_{i}$ can be chosen satisfying (7) so that all the integrals on the r.h.s. of (6) are finite.

Now

$$
\int_{\mathscr{Z}}\left|\frac{\partial^{k_{i}} \theta_{l}}{\partial x_{j}^{k_{i}}}(x)\right|^{2 q_{i}} d x \leq\left[M(s, \mathscr{Z})\left\|\theta \mid W_{2}^{s}\right\|\right]^{2}<\infty
$$

provided $q_{i}>1$ and

$$
k_{i}-d / 2 q_{i} \leq s-d / 2
$$

(use $W_{2 q_{i}}^{k_{i}}=B_{2 q_{i}, 2 q_{i}}^{k_{i}} \supset B_{2 q_{i}, 2}^{k_{i}} \supset B_{22}^{s}=W_{2}^{s}$, which follow from (8) through (12), and the fact that $W_{2 q_{i}}^{k_{i}}$ norm squared dominates the integral in (13)), which is equivalent to

$$
1<q_{i} \leq d /\left[2\left(k_{i}-s\right)+d\right] \text {. }
$$

Note that $k_{i}-s<0$ since $k_{i} \leq k<s$ and that $d+2\left(k_{i}-s\right)>0$ since $k_{i} \geq 1$ and $s<d / 2+1$, so the last member of (14) is greater than 1. Also,

$$
\begin{aligned}
& \int_{|h| \leq \delta}|h|^{-2(s-k)-d}\left\{\int_{\mathscr{Z}(h, \varepsilon, \tau)}\left|\frac{\partial^{k_{m}} \theta_{l}}{\partial x_{j}^{k_{m}}}(x+h)-\frac{\partial^{k_{m}} \theta_{l}}{\partial x_{j}^{k_{m}}}(x)\right|^{2 q_{m}} d x\right\}^{1 / q_{m}} d h \\
& \leq\left[M(s, \mathscr{X})\left\|\theta \mid W_{2}^{s}\right\|\right]^{2}<\infty
\end{aligned}
$$

provided $q_{m}>1$ and

$$
k_{m}+(s-k)-d / 2 q_{m} \leq s-d / 2
$$

(use $B_{2 q_{m}, 2}^{k_{m}+(s-k)} \supset B_{22}^{s}=W_{2}^{s}$, and the equivalent norm in (1)), which is equivalent to

$$
1<q_{m} \leq d /\left[2\left(k_{m}-k\right)+d\right]
$$

Note that $k_{m}<k$ since there is more than 1 summand in (4), so the denominator in the last member of (16) is less than $d$, and it is positive since $k_{m} \geq 1$ and $k \leq d / 2$, so the last member of (16) is greater than 1. (Note that if there is only 1 summand in (4) then finiteness of (6) is trivial since it is then one of the terms in the equivalent norm (1) for $\left\|\theta \mid W_{2}^{s}\right\|$.) To finish the proof that all integrals on the r.h.s. of (6) are finite and, in fact, that the whole r.h.s. of (6) is dominated by a constant (depending only on $s, \mathscr{Z}, H$, and $R_{0}$ ) multiple of $\left\|\theta \mid W_{2}^{s}\right\|^{2}$, it suffices to show we can find $q_{i}$ 's satisfying (7), (14), and (16), which is possible if

$$
1 \geq\left[d-2\left(k-k_{m}\right)\right] / d+\sum_{1 \leq i \neq m}\left[2\left(k_{i}-s\right)+d\right] / d .
$$

Now the r.h.s. is equal to

$$
\begin{aligned}
(2 / d) & {\left[\sum_{i} k_{i}-(s-d / 2) \sum_{i} 1+(s-k)\right] } \\
= & (2 / d)\left[k-(s-d / 2) \sum_{i} 1+(s-k)\right] \\
& =(2 / d)\left[s-(s-d / 2) \sum_{i} 1\right] \leq(2 / d)[s-(s-d / 2)] \leq 1,
\end{aligned}
$$


so we may find $q_{i}$ 's such that (6) is bounded by a constant (depending on $s$, $\mathscr{Z}, H$, and $R_{0}$ ) multiple of $\left\|\theta \mid W_{2}^{s}\right\|$.

Finally, we need to consider integrals of the form

$$
\begin{aligned}
& \int_{|h| \leq \delta}|h|^{-2(s-k)-d} \int_{\mathscr{Z}(h, \varepsilon, \tau)}\left\{\left[\Delta_{h} \frac{\partial^{k_{0}} H}{\partial t_{l}^{k_{0}}}(x, \theta(x))\right] \prod_{i \geq 1} \frac{\partial^{k_{i}} \theta_{l}}{\partial x_{j}^{k_{i}}}(x)\right\}^{2} d x d h \\
& \leq \int_{|h| \leq \delta}|h|^{-2(s-k)-d}\left\{\int_{\mathscr{Z}(h, \varepsilon, \tau)}\left|\Delta_{h} \frac{\partial^{k_{0}} H}{\partial t_{l}^{k_{0}}}(x, \theta(x))\right|^{2 q_{0}} d x\right\}^{1 / q_{0}} d h \\
& \quad \times \prod_{i \geq 1}\left\{\int_{\mathscr{X}}\left|\frac{\partial^{k_{i}} \theta_{l}}{\partial x_{j}^{k_{i}}}(x)\right|^{2 q_{i}} d x\right\}^{1 / q_{i}}
\end{aligned}
$$

where

$$
1 / q_{0}+\sum_{i \geq 1} 1 / q_{i} \leq 1
$$

and (14) holds for all $i \geq 1$, which implies that the product of integrals over $i \geq 1$ on the r.h.s. of (17) is finite. For the other factor, apply the mean value theorem to show it is equal to

$$
\begin{gathered}
\int_{|h| \leq \delta}|h|^{-2(s-k)-d}\left\{\int_{\mathscr{Z}(h, \varepsilon, \tau)} \mid \sum_{j=1}^{d} \frac{\partial^{k_{0}+1} H}{\partial x_{j} \partial t_{l}^{k_{0}}}(x+\alpha h, \alpha \theta(x+h)+(1-\alpha) \theta(x)) h_{j}\right. \\
\left.+\left.\sum_{j=1}^{q} \frac{\partial^{k_{0}+1} H}{\partial t_{j} \partial t_{l}^{k_{0}}}(x+\alpha h, \alpha \theta(x+h)+(1-\alpha) \theta(x)) \Delta_{h} \theta_{j}(x)\right|^{2 q_{0}} d x\right\}^{1 / q_{0}} d h
\end{gathered}
$$

where $\alpha=\alpha(x, \theta(x), h)$ is between 0 and 1 . Since $\partial^{k_{0}+1} H / \partial x_{j} \partial t_{0}^{k_{0}}$ is bounded and $\left|h_{j}\right| \leq|h|$, it is easy to see that the first summation above after the integrations is bounded by a constant depending on $s, \mathscr{X}, H$, and $R_{0}$. For the second summation, we may use boundedness of $\partial^{k_{0}+1} H / \partial t_{j} \partial t_{l}^{k_{0}}$ to pull that factor out, and then we must deal with terms of the form

$$
\int_{|h| \leq \delta}|h|^{-2(s-k)-d}\left\{\int_{\mathscr{L}(h, \varepsilon, \tau)}\left|\Delta_{h} \theta_{j}(x)\right|^{2 q_{0}} d x\right\}^{1 / q_{0}} d h \leq\left\|\theta_{j} \mid B_{2 q_{0}, 2}^{(s-k)}\right\|^{2}
$$

where the latter inequality follows from (1). Now $B_{2 q_{0}, 2}^{(s-k)} \supset B_{22}^{s}$ if $1<q_{0}$ and

$$
(s-k)-d / 2 q_{0} \leq s-d / 2 \text {, }
$$

which is equivalent to

$$
1<q_{0} \leq d /[d-2 k]
$$

Since $d>1$, this latter pair of inequalities is possible. We also need from (18) that

$$
1 / q_{0} \leq 1-(2 / d)\left[k-(s-d / 2) \sum_{i \geq 1} 1\right] .
$$


One can check that (21) and (22) are compatible, so we can find the $q_{i}$ 's with the requisite properties.

Since the r.h.s. of (1) can be bounded by a linear combination of terms of the form of (17), (6), (5), and $\left\|H \mid C\left(\mathscr{X} \times S\left(R_{0}, \mathbb{R}^{q}\right)\right)\right\|$ (for the first term on the r.h.s. of (1)), the result follows.

Finally we briefly indicate the $d=1$ case. Here, $k=0$, so no chain rule is involved. One uses the mean value theorem in (1) similar to the last step above but then proceeds directly to $\left\|\theta \mid W_{2}^{s}\right\|$ since no Hölder step is used. This completes the proof.

\section{REFERENCES}

1. R. Adams, Sobolev spaces, Academic Press, New York, 1975.

2. D. D. Cox and F. O'Sullivan, Penalized likelihood-type estimators for generalized nonparametric regression, Technical Report, Department of Statistics, University of Washington, 1993; J. Multivar. Anal., submitted.

3. H. Triebel, Interpolation theory function spaces, differential operators, North-Holland, New York, 1978.

Department of Statistics, Rice University, P. O. Box 1892, Houston, TeXas 77251

E-mail address: dcox@rice.edu

Departments of Statistics and Biostatistics, University of Washington, Seattle, WASHINGTON 98195

E-mail address: finbarrestat.washington.edu 